\title{
Влияние глубоких уровней дислокаций в гетероэпитаксиальных InGaAs/GaAs и GaAsSb/GaAs $p-i-n$-структурах на время релаксации неравновесных носителей
}

\author{
(С) М.М. Соболев, Ф.Ю. Солдатенков
}

Физико-технический институт им. А.Ф. Иоффе Российской академии наук, 194021 Санкт-Петербург, Россия

E-mail: m.sobolev@mail.ioffe.ru

(Получена 5 июля 2017 г. Принята к печати 12 июля 2017 г.)

\begin{abstract}
Представлены результаты экспериментальных исследований вольт-фарадных характеристик, спектров нестационарной спектроскопии глубоких уровней $p^{+}-p^{0}-i-n^{0}$-гомоструктур на основе нелегированных бездислокационных слоев GaAs, и гетероструктур InGaAs/GaAs и GaAsSb/GaAs с однородными сетками дислокаций несоответствия, выращенных методом жидкофазной эпитаксии. Для структуры на основе GaAs в эпитаксиальных $p^{0}$ - и $n^{0}$-слоях были обнаружены дефекты акцепторного типа с глубокими уровнями, идентифицируемые как $H L 2$ и $H L 5$. B InGaAs/GaAs и $\mathrm{GaAsSb} / \mathrm{GaAs}$ гетероструктурах были выявлены электронные и дырочные глубокие уровни дислокаций, обозначаемые соответственно как $E D 1$ и $H D 3$. Параметры дырочных ловушек: энергии термической активации $\left(E_{t}\right)$, сечения захвата $\left(\sigma_{n}\right)$ и концентрации $\left(N_{t}\right)$, определенные из зависимостей Аррениуса, были соответственно $E_{t}=845 \mathrm{MэB}, \sigma_{p}=1.33 \cdot 10^{-12} \mathrm{~cm}^{2}, N_{t}=3.80 \cdot 10^{14} \mathrm{~cm}^{-3}$ для $\mathrm{InGaAs} / \mathrm{GaAs}$ и $E_{t}=848$ мэВ, $\sigma_{p}=2.73 \cdot 10^{-12} \mathrm{~cm}^{2}, N_{t}=2.40 \cdot 10^{14} \mathrm{~cm}^{-3}$ для $\mathrm{GaAsSb} / \mathrm{GaAs}$. Были проведены оценки времен релаксации концентраций неравновесных носителей при участии в этом процессе глубоких акцепторных ловушек, связанных с дислокациями. Они оказались равными для InGaAs/GaAs гетероструктур $2 \cdot 10^{-10} \mathrm{c}$, для $\mathrm{GaAsSb} / \mathrm{GaAs}$ гетероструктур $1.5 \cdot 10^{-10} \mathrm{c}$, для GaAs гомоструктуры $1.6 \cdot 10^{-6} \mathrm{c}$.
\end{abstract}

DOI: $10.21883 /$ FTP.2018.02.45440.8680

\section{1. Введение}

Существует неослабевающий интерес к арсениду галлия как материалу для создания высоковольтных и высокотемпературных быстродействующих $p-i-n$-структур. К настоящему времени для создания высоковольтных GaAs $p-i-n$-структур в основном используется метод жидкофазной эпитаксии (ЖФЭ) из ограниченного раствора-расплава $\mathrm{Ga}-\mathrm{As}$ с контролируемым распределением остаточных примесей [1-4]. Как было отмечено в работе [5], существенное влияние на временны́е характеристики арсенид-галлиевых диодов (время жизни неравновесных носителей заряда и нарастания перепадов напряжения), а также на блокируемое диодными структурами напряжение должны оказывать дефекты и интерфейсные состояния с глубокими уровнями (ГУ), образующиеся в процессе эпитаксиального роста $p-i-n$-структур. К настоящему времени известны способы управления ансамблем собственных дефектов в эпитаксиальных слоях GaAs, полученного методом ЖФЭ: во-первых, через изменение температур начала кристаллизации $\left(T_{b}\right)$ [6], во-вторых, за счет изменения атомной доли изовалентной примеси висмута Ві в составе растворителя $(\mathrm{Ga}+\mathrm{Bi})$, которое должно приводить к изменению отношения концентрации Ga и As [7]. $\mathrm{B} p^{+}-p^{0}-i-n^{0}$-структурах GaAs, выращенных при температурах начала кристаллизации $T_{b}=650-800^{\circ} \mathrm{C}$, в спектрах нестационарной спектроскопии глубоких уровней (DLTS) были обнаружены HL5 и HL2 дырочные ловушки [8], характерные для слоев GaAs, полученных методом ЖФЭ в атмосфере водорода [6]. При повышении температуры начала кристаллизации $T_{b} \geq 850^{\circ} \mathrm{C}$ в эпитаксиальных слоях GaAs, помимо HL5 и HL2 дефектов, наблюдалось образование дефекта, подобного EL2 [6,9]. C увеличением содержания $\mathrm{Bi}$ в растворе-расплаве происходило уменьшение концентрации ГУ дефектов $H L 5$ и $H L 2$, связанное с изменением отношения концентрации $\mathrm{Ga}$ и $\mathrm{As}$ [7]. Для эпитаксиальных слоев $\mathrm{In}_{x} \mathrm{Ga}_{1-x} \mathrm{As}$ и $\mathrm{GaAs}_{1-x} \mathrm{Sb}_{x}(x<0.012$ мол\%), выращенных на GaAs-подложке, наблюдали аналогичное подавление дырочных ловушек при одновременном уменьшении плотности дислокаций $[10,11]$. Авторы работы [12] на основании полученных экспериментальных результатов пришли к выводу, что обнаруженные эффекты подавления связаны с локальными напряжениями, возникающими вокруг атомов In и $\mathrm{Sb}$ в твердых растворах $\mathrm{In}_{x} \mathrm{Ga}_{1-x} \mathrm{As}$ и $\mathrm{GaAs}_{1-x} \mathrm{Sb}_{x}$. В работах $[13,14]$ отмечалось, что при увеличении содержания изовалентных примесей в твердых растворах до $x \approx(0.2-3$ мол\%) из-за различия в параметрах решетки подложки и слоя при превышении толщины слоя выше критического значения происходит формирование двухмерной сетки $60^{\circ}$ дислокаций несоответствия в интерфейсе подложка-слой. Их появление может сопровождаться формированием прорастающих через эпитаксиальный слой дислокаций. В работах $[14,15]$, используя технику нестационарной спектроскопии глубоких уровней (DLTS) в гетероструктурах $\operatorname{In}_{x} \mathrm{Ga}_{1-x} \mathrm{As} / \mathrm{GaAs}$ и $\mathrm{GaAs}_{1-x} \mathrm{Sb}_{x} / \mathrm{GaAs}$, выращенных методом ЖФЭ, были обнаружены глубокие уровни $(Г У)$ с энергией в запретной зоне $\left(E_{V}+0.67\right.$ эВ $)$ и $\left(E_{C}-0.64\right.$ эВ). Эти уровни приписывались соответственно дислокациям несоответствия и прорастающим 
дислокациям и могли действовать как центры рекомбинации или ловушки для свободных носителей. Ранее в работе [4] было показано, что появление однородной сетки дислокаций несоответствия типично для слоев $\mathrm{In}_{x} \mathrm{Ga}_{1-x} \mathrm{As}$ толщиной от 50 до 65 мкм с составом $x$ в диапазоне от 1.5 до 3 мол\%. Типичное значение плотности дислокаций для таких сеток составляло величину порядка $100 \mathrm{~cm}^{-1}$. С появлением и развитием структурных дефектов такого типа связывались наблюдаемые эффекты значительного уменьшения времени релаксации неравновесных носителей заряда и плавного снижения блокируемых высоковольтными диодными $\mathrm{InGaAs} / \mathrm{GaAs}$ гетероструктурами напряжений, что обусловливалось постепенным увеличением плотности дислокационной сетки при повышении $x$ до $\sim 3$ мол\% [4]. Таким образом, возникает необходимость проведения работ по выявлению и изучению ансамбля ГУ в гетероструктурах $\mathrm{In}_{x} \mathrm{Ga}_{1-x} \mathrm{As} / \mathrm{GaAs}$ и $\mathrm{GaAs}_{1-x} \mathrm{Sb}_{x} / \mathrm{GaAs}$ с дислокациями несоответствия и их влияния на времена релаксации неравновесных носителей заряда.

В данной работе приведены результаты сравнительных исследований ГУ, связанных с точечными дефектами и дислокациями, в гомоэпитаксиальных слоях $\mathrm{GaAs}$ и гетероэпитаксиальных слоях $\mathrm{InGaAs} / \mathrm{GaAs}$ и $\mathrm{GaAsSb} / \mathrm{GaAs} p^{0}-i-n^{0}$-диодов, изготовленных методом ЖФЭ, с помощью методов емкостной спектроскопии: вольт-фарадного $(C-V)$ и нестационарной спектроскопии глубоких уровней (DLTS).

\section{2. Экспериментальные образцы}

Изготовление GaAs, $\operatorname{In}_{x} \mathrm{Ga}_{1-x} \mathrm{As} / \mathrm{GaAs}$ и $\mathrm{GaAs}_{1-x} \mathrm{Sb}_{x} / \mathrm{GaAs} p^{+}-p^{0}-i-n^{0}-n^{+}$-структур проводилось в два этапа с помощью метода ЖФЭ. Эпитаксиальное выращивание высоковольтных слабо легированных плавных $p^{0}-i-n^{0}$-переходов $\mathrm{GaAs}$, InGaAs и $\mathrm{GaAsSb}$ проводилось на подложках $p$-GaAs с ориентацией (111), легированных цинком до $\sim 5 \cdot 10^{18} \mathrm{~cm}^{-3}$, из ограниченного $\mathrm{Ga}-\mathrm{As}$, In- $\mathrm{Ga}-\mathrm{As}$ или $\mathrm{Sb}-\mathrm{Ga}-\mathrm{As}$ расплава в кварцевой кассете в атмосфере водорода от $850^{\circ} \mathrm{C}$ до комнатной температуры. При таком способе выращивания $p^{0}-i-n^{0}$-структур возможно получение толстых (протяженных) слабо легированных компенсированных $i$-областей с концентрацией свободных носителей порядка $10^{13}-10^{14} \mathrm{~cm}^{-3}$, что позволяет достичь блокирующих диодами напряжений $U_{b}$ до $2000 \mathrm{~B}$ [16]. Сильно легированные теллуром $n^{+}$-GaAs слои эмиттеров диодных структур выращивались в графитовой поршневой кассете. Типичное распределение концентрации свободных носителей заряда по толщине GaAs или InGaAs $p-i-n$-структур и описание методики изготовления таких структур представлены в $[2,4]$. Профили распределения свободных носителей заряда получали при послойном стравливании структуры из вольт-емкостных зависимостей обратносмещенного барьера Шоттки с помощью ртутного зонда. Образцы диодов (чипы) имели форму мезаструктур, которые получали путем химического травления активных слоев до подложки. Ha $p^{+}$-GaAs подложку наносили многослойный контакт $\mathrm{AgMn}-\mathrm{Ni}-\mathrm{Au}$, а на $n^{+}$-GaAs эмиттер - $\mathrm{AuGe}-\mathrm{Ni}-\mathrm{Au}$.

Для исследования реальной кристаллической структуры слабо легированных слоев в гетероструктурах $\mathrm{In}_{x} \mathrm{Ga}_{1-x} \mathrm{As} / \mathrm{GaAs}$ использовалось $\mathrm{Cu} K_{\alpha}$-излучение и асимметричные отражения 422, 533, 331 в рентгенотопографическом методе обратного отражения с разной глубиной информационного слоя. Данная методика изучения кристаллической структуры слоев изложена в [17].

\section{3. Результаты и обсуждение}

Были проведены исследования вольт-фарадных $(C-V)$ характеристик и DLTS-спектров чипов $p^{+}-p^{0}-i-n^{0}-$ структур на основе гомоэпитаксиального GaAs и гетероэпитаксиальных слоев $\operatorname{In}_{x} \mathrm{Ga}_{1-x} \mathrm{As} / \mathrm{GaAs}$ и $\mathrm{GaAs}_{1-x} \mathrm{Sb}_{x} / \mathrm{GaAs}$ с помощью DLTS спектрометра (DL4600 фирмы BIORAD (Англия)) с Boxcar интегратором. Исследования $C-V$-характеристик диодов проводили при различных температурах и условиях измерения: в темноте и при освещении образца белым светом в процессе измерения $C-V$-характеристик. Для удобства представления результатов $C-V$-измерений и их дальнейшей интерпретации, используя метод дифференциальной емкости [18], из $C-V$-характеристик рассчитывались профили распределения эффективной концентрации свободных носителей заряда $\left(n^{*}\right)$ по толщине $(W)$ слоя объемного заряда (CO3). Исследования $p^{+}-p^{0}-i-n^{0}$-структур на основе эпитаксиального $\mathrm{GaAs}$ показали, что наблюдаемые изменения $C-V$-характеристик связаны с температурным ходом диффузионного потенциала. Оптическая перезарядка глубоких ловушек акцепторного типа в $i$-слое и примыкающих к нему $p^{0}$ - и $n^{0}$-слоях не приводила к существенному изменению эффективной концентрации носителей в зоне проводимости эпитаксиальных слоев $\left(n^{*}\right)$. Толщина слоя объемного заряда $(W)$ при температуре измерения $300 \mathrm{~K}$ составила величину порядка 3.5 мкм. Для $C-V$-характеристик (профилей распределения $n^{*}(W)$ ) $p^{+}-p^{0}-i-n^{0}$-структур на основе гетероэпитаксиальных слоев $\operatorname{In}_{x} \mathrm{Ga}_{1-x} \mathrm{As} / \mathrm{GaAs}$ и $\mathrm{GaAs}_{1-x} \mathrm{Sb}_{x} / \mathrm{GaAs}$ выявились существенные отличия от структур на основе GaAs (рис. 1, $a, b)$. Результаты, приведенные для обеих гетероструктур на рис. $1, a, b$, показывают, что при понижении температуры измерения образца до $86 \mathrm{~K}$ наблюдается рост толщины СОЗ. Оказалось, что для этих структур величины приращения толщины СО3 при изменении температуры от 300 до $86 \mathrm{~K}$ существенно больше тех значений, которые были рассчитаны из предположений, что они связаны с температурным ходом изменения диффузионного потенциала. Также для обеих $p^{+}-p^{0}-i-n^{0}$-гетероструктур была выявлена зависимость $C-V$-характеристик от наличия оптической подсветки при измерении образца (рис. $1, a, b)$. Подсвет- 

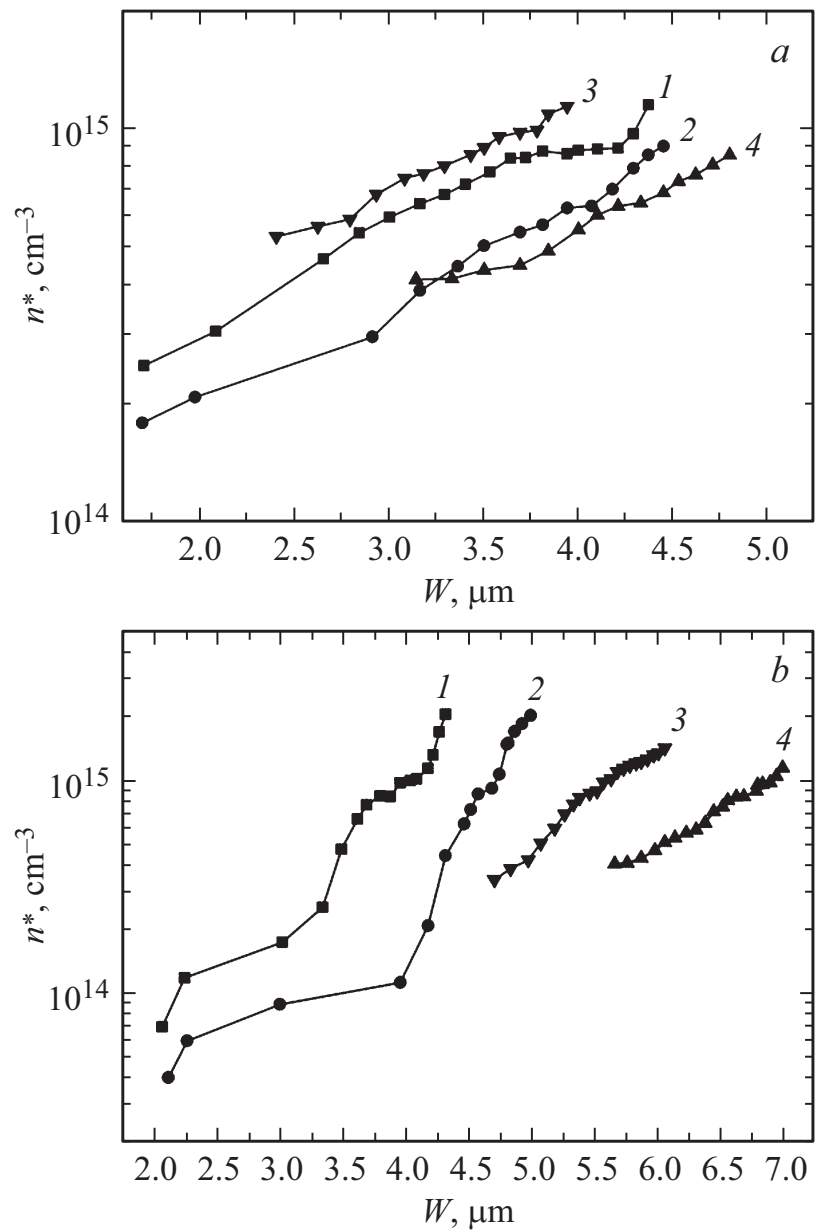

Рис. 1. Профили распределения эффективной концентрации свободных носителей заряда $n^{*}$ по толщине $W$ слоя объемного заряда $p^{+}-p^{0}-i-n^{0}$-диодов на основе гетероэпитаксиальных слоев: $a-\mathrm{In}_{x} \mathrm{Ga}_{1-x} \mathrm{As} / \mathrm{GaAs}$ и $b-\mathrm{GaAs}_{1-x} \mathrm{Sb}_{x} / \mathrm{GaAs}$, измеренных при различных температурах $T, \mathrm{~K}: 1,2-300$; $3,4-86$; в темноте $(2,4)$ и с подсветкой $(1,3)$.

ка структур приводила к уменьшению толщины СО3 как при 86, так и при $300 \mathrm{~K}$. Наиболее вероятной причиной таких изменений толщины СОЗ от температуры и подсветки могут быть интерфейсные глубокие состояния донорного и акцепторного типа на гетерограницах $p^{+}-p^{0}-i-n^{0}$-структур. Влияние плотности интерфейсных состояний $N_{s s}$ на $C-V$-характеристику $p-n$-перехода исследовалось подробно в работах [19-22]. Наличие интерфейсных состояний связывается с формированием на гетерограницах подложка-слой исследуемых структур дислокаций несоответствия.

Для всех трех типов $p^{+}-p^{0}-i-n^{0}$-диодных структур были измерены DLTS-спектры при различных значениях напряжений смещения $V_{r}$ и импульсов заполнения $V_{f}$ (рис. 2). Ранее было отмечено, что эпитаксиальные слои твердого раствора представляют собой плавный $p-i-n$-переход с концентрацией свободных носителей заряда в $i$-слое около $10^{13}-10^{14} \mathrm{~cm}^{-3}$. Поэтому
DLTS-спектры измерялись как при отрицательных, так и положительных значениях напряжений смещения $V_{r}$. При этом напряжения импульсов заполнения $V_{f}$ имели положительные значения, что позволяло выявлять глубокие ловушки основных и неосновных носителей в $p^{0}$ - и $n^{0}$-слоях исследуемых структур. На рис. 2 показаны DLTS-спектры GaAs $p^{+}-p^{0}-i-n^{0}$-структуры, измеренные для двух значений $V_{r}=-1.01$ (1 спектр) и $0.12 \mathrm{~B}$ (2 спектр) и напряжении импульса заполнения $V_{f}=0.96 \mathrm{~B}$. Видно, что при $V_{r}=0.12 \mathrm{~B}$ наблюдается четыре отрицательных DLTS-пика (рис. 2), связанных с ловушками основных носителей. Поскольку импульс заполнения и напряжение, при котором производилось измерение DLTS-сигнала, были приложены в прямом направлении, то заполнение ловушек носителями и их эмиссия должны происходить в основном из $p^{0}$-слоя. В этом слое основными носителями являются дырки, а захват и эмиссия определяются акцепторами с ГУ. В спектрах DLTS, кроме четырех отрицательных пиков, наблюдался один DLTS-пик с положительным знаком, появление которого в DLTS-спектре связано с эмиссией дырок с ГУ, расположенных в $n^{0}$-слое. При $V_{r}=-1.01$ В пики с отрицательным знаком либо совсем исчезли, либо их амплитуды существенно уменьшились, но появилось два DLTS-пика с положительными знаками и большой амплитудой: низкотемпературный и высокотемпературный (рис. 2), и представляющих ловушки неосновных носителей. Так как при $V_{r}=-1.01$ В напряжение, при котором производилось измерение DLTS-сигнала, было включено в обратном направлении, эмиссия неосновных носителей происходила из $n^{0}$-слоя и связывалась с дырками, которые были захвачены на глубокие состояния акцепторов. Эти измерения показали, что включение $V_{r}$ при двух различных значениях, с прямым и обратным напряжением смещения, позволило нам идентифицировать ГУ в $p^{0}$ - и $n^{0}$-слоях и показать, что

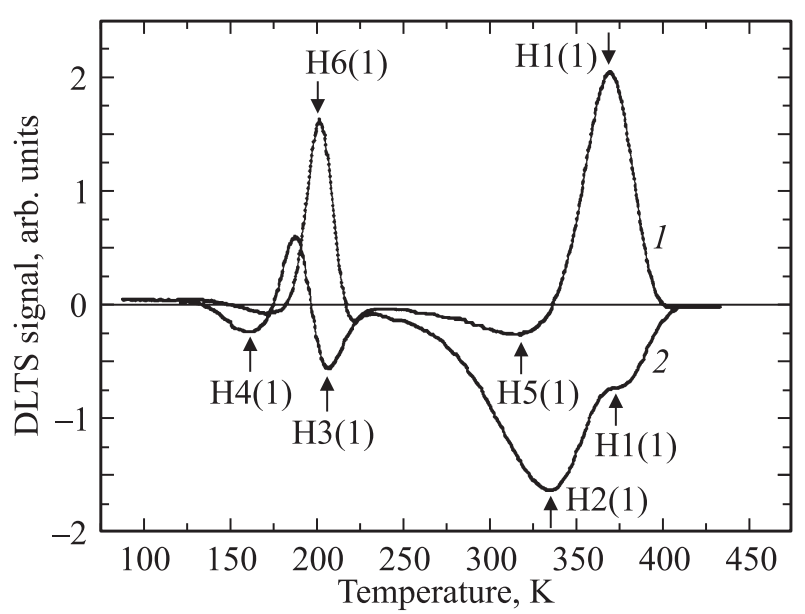

Рис. 2. DLTS-спектры GaAs $p^{+}-p^{0}-i-n^{0}$-диода с темпом окон $200^{-1}$ с, при напряжении импульса заполнения $V_{f}=0.96 \mathrm{~B}$ и напряжениях обратного смещения $V_{r}, \mathrm{~B}: 1-(-1.01)$, $2-+0.12$. 
Идентификация электронных и дырочных ловушек в $\mathrm{GaAs}, \operatorname{In}_{x} \mathrm{Ga}_{1-x} \mathrm{As} / \mathrm{GaAs}$ и $\mathrm{GaAs}_{1-x} \mathrm{Sb}_{x} / \mathrm{GaAs}^{+}-p^{0}-i-n-n^{+}$диодах, полученных методом ЖФЭ.

\begin{tabular}{|c|c|c|c|c|c|c|}
\hline $\begin{array}{c}\text { Номер } \\
\text { глубокого } \\
\text { уровня }\end{array}$ & $\begin{array}{c}\text { Энергия, } \\
\text { мэВ }\end{array}$ & $\begin{array}{c}\text { Сечение } \\
\text { захвата, } \text { см }^{2}\end{array}$ & $\begin{array}{c}\text { Концентрация ГУ, } \\
\mathrm{cm}^{-3}\end{array}$ & Идентификация & Природа & Ссылки \\
\hline \multicolumn{7}{|c|}{ GaAs } \\
\hline $\begin{array}{l}H 1(1) \\
H 2(1) \\
H 4(1) \\
H 5(1) \\
H 6(1)\end{array}$ & $\begin{array}{l}734 \\
499 \\
302 \\
643 \\
447\end{array}$ & $\begin{array}{l}1.05 \cdot 10^{-14} \\
4.69 \cdot 10^{-17} \\
1.05 \cdot 10^{-14} \\
2.06 \cdot 10^{-14} \\
5.38 \cdot 10^{-13}\end{array}$ & $\begin{array}{c}5.0 \cdot 10^{13} \\
8.84 \cdot 10^{15} \\
2.0 \cdot 10^{12} \\
4.0 \cdot 10^{12} \\
3.8 \cdot 10^{13}\end{array}$ & $\begin{array}{l}H L 2 \\
H L 3 \\
H L 6 \\
H L 9 \\
H L 5\end{array}$ & $\begin{array}{c}V_{\mathrm{Ga}}+? \\
\mathrm{Fe} \\
V_{\mathrm{Ga}}+?\end{array}$ & $\begin{array}{c}{[6-8]} \\
{[8]} \\
{[8]} \\
{[8]} \\
{[6-8]}\end{array}$ \\
\hline \multicolumn{7}{|c|}{ InGaAs } \\
\hline $\begin{array}{c}E 1(2) \\
H 1(2) \\
H 1^{*}(2) \\
H 6(2)\end{array}$ & $\begin{array}{l}653 \\
774 \\
845 \\
409 \\
\end{array}$ & $\begin{array}{l}1.06 \cdot 10^{-13} \\
1.73 \cdot 10^{-12} \\
1.33 \cdot 10^{-12} \\
6.35 \cdot 10^{-14}\end{array}$ & $\begin{array}{r}3.35 \cdot 10^{14} \\
3.8 \cdot 10^{14} \\
3.8 \cdot 10^{14} \\
3.2 \cdot 10^{13} \\
\end{array}$ & $\begin{array}{c}E D 1 \\
H D 3 \\
H D 3 \\
H L 5\end{array}$ & $V_{\mathrm{Ga}}+?$ & $\begin{array}{c}{[14,15]} \\
{[14,15]} \\
{[14,15]} \\
{[6-8]} \\
\end{array}$ \\
\hline \multicolumn{7}{|c|}{$\mathrm{GaAsSb}$} \\
\hline $\begin{array}{l}E 1(3) \\
H 1(3) \\
H 5(3) \\
H 6(3)\end{array}$ & $\begin{array}{l}683 \\
848 \\
627 \\
385\end{array}$ & $\begin{array}{l}1.16 \cdot 10^{-14} \\
2.73 \cdot 10^{-12} \\
1.69 \cdot 10^{-15} \\
3.95 \cdot 10^{-14}\end{array}$ & $\begin{array}{r}1.60 \cdot 10^{14} \\
2.39 \cdot 10^{14} \\
3.8 \cdot 10^{13} \\
1.98 \cdot 10^{13}\end{array}$ & $\begin{array}{l}E D 1 \\
H D 3 \\
H L 9 \\
H L 5\end{array}$ & $V_{\mathrm{Ga}}+?$ & $\begin{array}{c}{[14,15]} \\
{[14,15]} \\
{[8]} \\
{[6-8]}\end{array}$ \\
\hline
\end{tabular}

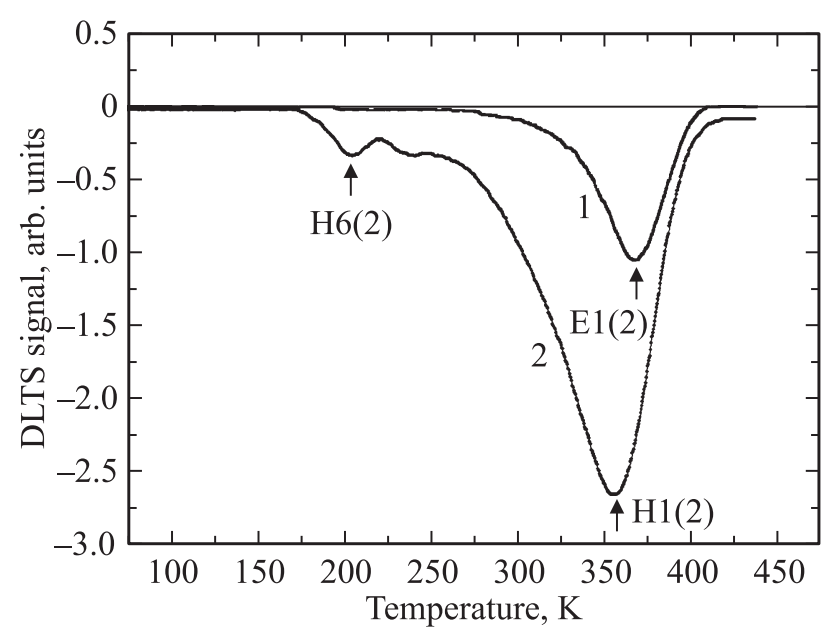

Рис. 3. DLTS-спектры InGaAs/GaAs $p^{+}-p^{0}-i-n^{0}$-диода, с темпом окон $200^{-1} \mathrm{c}$, при напряжении импульса заполнения $V_{f}=0.50 \mathrm{~B}$ и напряжениях обратного смещения $V_{r}, \mathrm{~B}$ : $1-(-2.70), 2-+0.01$.

они принадлежат одним и тем же дефектам, хорошо известным для GaAs, полученного методом ЖФЭ: HL2 и $H L 5$. Параметры акцепторных ГУ и их идентификация (см. таблицу) были определены с помощью зависимости Аррениуса. Подход, примененный выше для выявления дефектов с ГУ в $p^{+}-p^{0}-i-n^{0}$-структуре GaAs с помощью DLTS-спектров, был использован для исследования диодов $p^{+}-p^{0}-i-n^{0}$-гетероструктур InGaAs/GaAs и $\mathrm{GaAsSb} / \mathrm{GaAs}$, выращенных при $T_{b}=850^{\circ} \mathrm{C}$ (рис. 3,4).
По DLTS-спектрам, полученным при $V_{r}=-2.70 \mathrm{~B}$ и $V_{f}=0.50$ В для $\mathrm{InGaAs} / \mathrm{GaAs}$ и $\mathrm{GaAsSb} / \mathrm{GaAs}$ гетероструктур, в $n^{0}$-слоях было выявлено по одному отрицательному высокотемпературному DLTS-пику, связанному с эмиссией электронов из глубокой ловушки донорного типа. Энергии термической активации и сечения захвата этой ловушки с ГУ, определенные с помощью зависимости Аррениуса, были $E_{t}=653$ мэВ, $\sigma_{n}=1.06 \cdot 10^{-13} \mathrm{~cm}^{2}$ для $\mathrm{InGaAs} / \mathrm{GaAs}$ и $E_{t}=683 \mathrm{мэB}$,

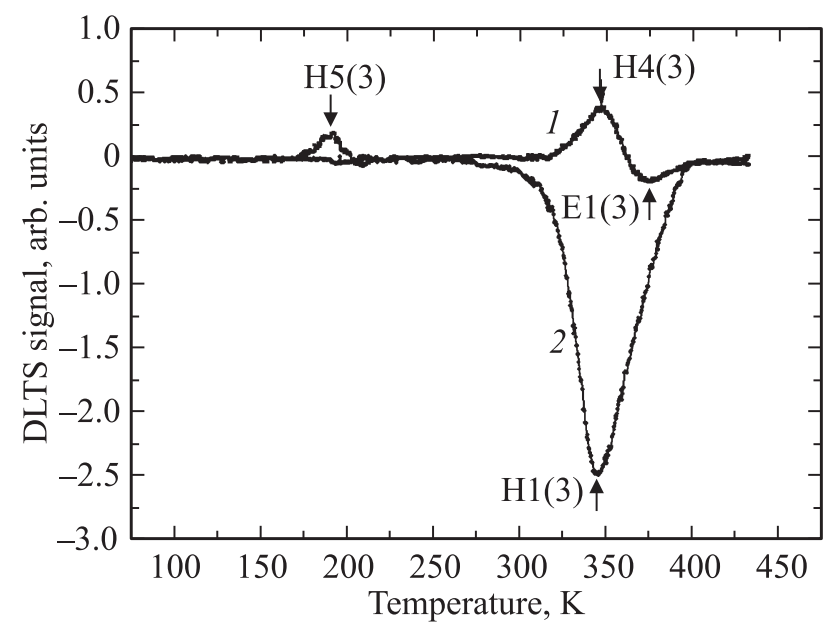

Рис. 4. DLTS-спектры $\mathrm{GaAsSb} / \mathrm{GaAs} p^{+}-p^{0}-i-n^{0}$-диода с темпом окон $200^{-1}$ c, при напряжении импульса заполнения $V_{f}=0.50 \mathrm{~B}$ и напряжениях обратного смещения $V_{r}, \mathrm{~B}$ : $1-(-2.70), 2-+0.01$. 
$\sigma_{n}=1.16 \cdot 10^{-14} \mathrm{~cm}^{2}$ для $\mathrm{GaAsSb} / \mathrm{GaAs}$ (см. таблицу). Концентрации электронных ловушек были соответственно: $3.35 \cdot 10^{14} \mathrm{~cm}^{-3}$ и $1.60 \cdot 10^{14} \mathrm{~cm}^{-3}$. Эти электронные ловушки близки по параметрам к глубокому уровню $E D 1$, который наблюдался в работах $[14,15]$ и связывался с прорастающими дислокациями, образующимися вблизи интерфейсов гетероструктур InGaAs/GaAs и $\mathrm{GaAsSb} / \mathrm{GaAs}$. B GaAs $p^{+}-p^{0}-i-n^{0}$-структуре электронные ловушки не проявлялись. Для выявления дефектов с ГУ в $p^{0}$-слоях исследуемых $\mathrm{InGaAs} / \mathrm{GaAs}$ и $\mathrm{GaAsSb} / \mathrm{GaAs}$ гетероструктур измерения DLTS-спектров проводились с $V_{r}=0.01 \mathrm{~B}$ и $V_{f}=0.50 \mathrm{~B}$. Для обеих гетероструктур в DLTS-спектрах $p^{0}$-слоя наблюдался отрицательный высокотемпературный пик, связанный в этом случае уже с эмиссией дырок из глубокой ловушки акцепторного типа. Параметры этой ловушки с ГУ были соответственно: $E_{t}=774$ мэВ и $\sigma_{p}=1.23 \cdot 10^{-13} \mathrm{~cm}^{2}$ для $\mathrm{InGaAs} / \mathrm{GaAs}$ и $E_{t}=848$ мэВ и $\sigma_{p}=2.73 \cdot 10^{-12} \mathrm{~cm}^{2}$ для $\mathrm{GaAsSb} / \mathrm{GaAs}$ (см. таблицу). Концентрации дырочных ловушек были соответственно: $3.80 \cdot 10^{14}$ и $2.40 \cdot 10^{14} \mathrm{~cm}^{-3}$. При измерении DLTS-спектра InGaAs/GaAs гетероструктуры с $V_{r}=0.01 \mathrm{~B}$ и $V_{f}=1.00 \mathrm{~B}$ произошло изменение параметров высокотемпературной дырочной ловушки при неизменной ее концентрации, - они стали равными $E_{t}=845$ мэВ и $\sigma_{p}=1.33 \cdot 10^{-12} \mathrm{~cm}^{2}$ (см. таблицу). Они оказались подобными тем значениям, которые были определены для дырочной ловушки в $\mathrm{GaAsSb} / \mathrm{GaAs}$ гетероструктуре. Сравнение зависимостей Аррениуса дырочных ловушек с ГУ (рис. 5), обнаруженных в $p^{0}$-слоях трех типов исследуемых с помощью метода DLTS $p^{+}-p^{0}-i-n^{0}$-структур, показывает, что это не дефекты, подобные $H L 2$, которые образуются в эпитаксиальных слоях GaAs. В работе $[14,15]$ было сделано сообщение об обнаружении в эпитаксиальных слоях $\mathrm{GaAs}_{1-x} \mathrm{Sb}_{x}$, полученных методом ЖФЭ, наряду с электронной ловушкой $E D 1$, дырочной ловушки HD3 с активационной энергией около 0.74 эВ. Эта дырочная ловушка приписывалась дислокациям несоответствия. В наших DLTS-измерениях InGaAs/GaAs гетероструктур была обнаружена зависимость энергии термической активации и сечения захвата акцепторных ловушек дислокации от импульса заполнения. Подобная зависимость может быть связана с существованием энергетической зоны в запрещенной зоне. На возможность существования подобной зоны было обращено внимание в монографии [23], где предлагалось рассматривать дислокацию как одномерный кристалл. Кроме того, для дислокаций, как было отмечено в монографии Матаре [24], характерны большие сечения захвата для дырок, что мы и наблюдали при DLTS-исследованиях обеих гетероструктур InGaAs/GaAs и GaAsSb/GaAs. Для дислокационных центров связь с решеткой посредством фононов по отношению к точечным дефектам уменьшается, такие центры с уровнями, расположенными посредине запрещенной зоны, могут быть эффективными центрами рекомбинации с равным числом партнеров из валентной

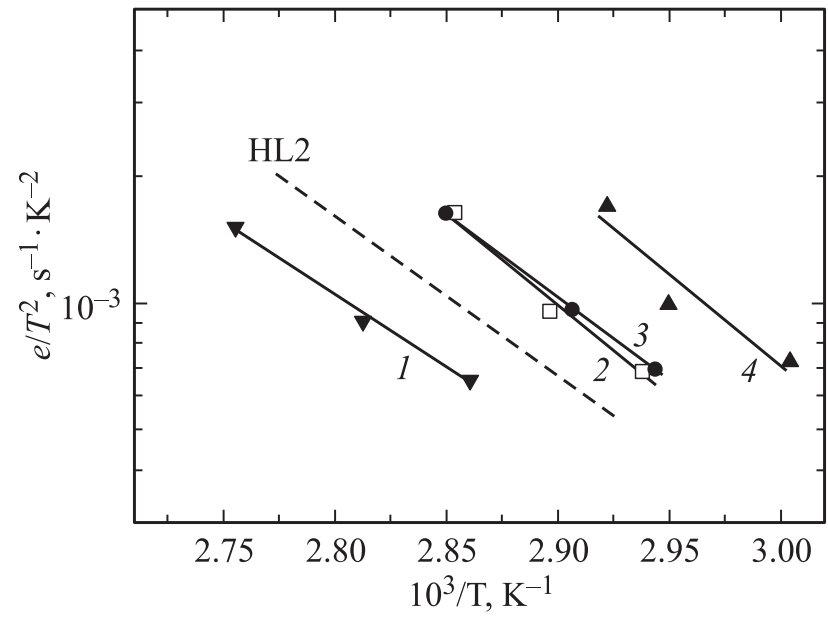

Рис. 5. Температурные зависимости Аррениуса темпов тепловой эмиссии дырок из глубоких акцепторных ловушек: $1-H 1(1), 2-H 1(2), 3-H 1^{*}(2), 4-H 1(3)$ в $p^{+}-p^{0}-i-n^{0}$-структурах на основе гомоэпитаксиальных слоев GaAs (1), гетероэпитаксиальных слоев $\operatorname{In}_{x} \mathrm{Ga}_{1-x} \mathrm{As} / \mathrm{GaAs}(2,3)$ и $\mathrm{GaAs}_{1-x} \mathrm{Sb}_{x} / \mathrm{GaAs}$ (4). Пунктирная линия показывает зависимость Аррениуса для $H L 2$ ловушки, идентифицированной в работе [8].

зоны и зоны проводимости [24]. Если предположить в таком случае, что сечение захвата электрона на нейтральный дислокационный центр незначительно отличается от сечения захвата дырки на отрицательно заряженный центр, то появляется возможность провести оценки времен релаксации концентраций неравновесных носителей. При этом значения основных параметров дислокационных ловушек с ГУ (энергии термической активации и их концентраций) мы определили выше. В общем виде соотношения, устанавливающие связь между величинами времен релаксации неравновесных носителей, сложным образом зависят от коэффициентов захвата ловушками электронов и дырок $C_{n}=\sigma_{n} \cdot v$ и $C_{p}=\sigma_{p} v$ (где $v$ - тепловая скорость) и энергий уровней [23]. Вид их может быть упрощен для специальных случаев, например для глубоких акцепторных уровней при низком уровне возбуждения, высокой концентрации ловушек, низкой температуре и выполнении условий [23]

$$
N_{t}^{0} \gg n_{0}+n_{1}, \quad N_{t}^{-} \ll p_{0}+p_{1} .
$$

При этом соотношения для времени релаксации неравновесных носителей упрощаются и имеют вид, показанный далее [23]:

$$
\frac{1}{\tau_{1}} \cong C_{n} \cdot N_{t}^{0}, \quad \frac{1}{\tau_{2}} \cong C_{p} \cdot N_{t}^{-} .
$$

Здесь $n_{1}$ и $p_{1}$ - концентрации электронов и дырок в зоне проводимости и валентной зоне соответственно, когда уровень Ферми совпадает с энергиями ГУ $\left(E_{t}\right) ; n_{0}$ и $p_{0}-$ равновесные концентрации электронов и дырок; $N_{t}^{0}$ - концентрация пустых акцептор- 
ных глубоких ловушек, $N_{t}^{-}-$концентрация заполненных электронами акцепторных глубоких ловушек. Оценим время релаксации неравновесных дырок для ловушки акцепторного типа, расположенной в верхней половине запретной зоны в $p^{0}$-слое в предположении, что она заполнена электронами. Для InGaAs/GaAs

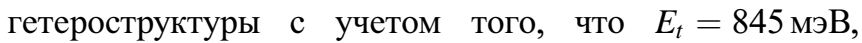
$\sigma_{p}=1.33 \cdot 10^{-12} \mathrm{~cm}^{2}, N_{t}^{-}=3.80 \cdot 10^{14} \mathrm{~cm}^{-3}$ и тепловая скорость $v=10^{7} \mathrm{~cm} / \mathrm{c}$, время релаксации $\tau_{2}=2 \cdot 10^{-10} \mathrm{c}$. Для $\mathrm{GaAsSb} / \mathrm{GaAs}$ гетероструктуры с $E_{t}=848$ мэВ, $\sigma_{p}=2.73 \cdot 10^{-12} \mathrm{~cm}^{2}$ и $N_{t}^{-}=2.40 \cdot 10^{14} \mathrm{~cm}^{-3}$ время релаксации $\tau_{2}=1.5 \cdot 10^{-10} \mathrm{c}$. Полагая, что сечение захвата электрона на нейтральный дислокационный центр $\sigma_{n}$ может иметь величину $\approx 10^{-13} \mathrm{~cm}^{2}$, тогда время релаксации $\tau_{1}$, согласно соотношению $(2)$, будет $\approx 10^{-9} \mathrm{c}$. Эффективные времена жизни неравновесных носителей заряда в базовых областях этих гетероструктур, определяемые методами Лэкса [25] или Госсика [26], были на уровне единиц наносекунд, что согласуется с нашими оценками, предполагающими, что релаксация концентрации неравновесных носителей определяется дислокационными центрами акцепторного типа. Аналогичные оценки, сделанные для GaAs $p^{+}-p^{0}-i-n^{0}$ структуры, в которой для $p^{0}$-слоя была выявлена дырочная ловушка с $E_{t}=695 \mathrm{MэB}, \sigma_{p}=3.89 \cdot 10^{-15} \mathrm{~cm}^{2}$ и $N_{t}^{-}=1.60 \cdot 10^{13} \mathrm{~cm}^{-3}$, показали время релаксации $\tau_{2}=1.6 \cdot 10^{-6}$ c. Это существенно больше, чем для $\mathrm{InGaAs} / \mathrm{GaAs}$ и $\mathrm{GaAsSb} / \mathrm{GaAs}$ гетероструктур.

\section{4. Заключение}

Были проведены исследования $C-V$-характеристик и DLTS-спектров $p^{+}-p^{0}-i-n^{0}$-гомоструктур на основе нелегированных бездислокационных слоев GaAs и гетероструктур InGaAs/GaAs и GaAsSb/GaAs с однородными сетками дислокаций несоответствия, выращенных методом ЖФЭ при температурах начала кристаллизации $850^{\circ} \mathrm{C}$. Было установлено, что все три исследуемые структуры представляли собой плавный $p-i-n$-переход с концентрацией свободных носителей заряда в $i$-слое $\approx 10^{13}-10^{14} \mathrm{~cm}^{-3}$, что позволило провести исследования DLTS-спектров в $p^{0}$ - и $n^{0}$-слоях $p^{0}-i-n^{0}$-структур, включая $V_{r}$ при двух различных значениях, с прямым и обратным напряжением смещения. Эти измерения позволили нам идентифицировать ГУ дефектов $H L 2$ и HL5 в $p^{0}$ - и $n^{0}$-эпитаксиальных слоях $\mathrm{GaAs}$, которые хорошо известны для GaAs, полученного методом ЖФЭ. Для гетероструктур InGaAs/GaAs и GaAsSb/GaAs было обнаружено наличие интерфейсных глубоких состояний на гетерограницах $p^{+}-p^{0}-i-n^{0}$-структур, которые проявляются при оптической подсветке для различных температур измерения $C-V$-характеристик. Измерения DLTS-спектров, проведенные для этих $p^{+}-p^{0}-i-n^{0}$-гетероструктур, позволили выявить в $n^{0}$ - и $p^{0}$-слоях электронные $E D 1$ и дырочные $H D 3$ ловушки, приписываемые соответственно дислокациям несоответствия и прорастающим дислокациям. Параметры глубокой ловушки электронного типа были $E_{t}=653 \mathrm{мэB}, \sigma_{n}=1.06 \cdot 10^{-13} \mathrm{~cm}^{2}, N_{t}=3.35 \cdot 10^{14} \mathrm{~cm}^{-3}$ для $\mathrm{InGaAs} / \mathrm{GaAs}$ и $E_{t}=683$ мэB, $\sigma_{n}=1.16 \cdot 10^{-14} \mathrm{~cm}^{2}$, $N_{t}=1.60 \cdot 10^{14} \mathrm{~cm}^{-3}$ для GaAsSb/GaAs. Параметры глубокой ловушки акцепторного типа, определенные из DLTS-спектров, полученных при $V_{r}=0.01 \mathrm{~B}$ и $V_{f}=$ $=0.50 \mathrm{~B}$, были соответственно: $E_{t}=774 \mathrm{мэB}, \sigma_{p}=$ $=1.23 \cdot 10^{-13} \mathrm{~cm}^{2}$ и $N_{t}=3.80 \cdot 10^{14} \mathrm{~cm}^{-3}$ для InGaAs/GaAs и $E_{t}=848$ мэВ, $\sigma_{p}=2.73 \cdot 10^{-12} \mathrm{~cm}^{2}$ и $N_{t}=2.40 \cdot 10^{14} \mathrm{~cm}^{-3}$ для $\mathrm{GaAsSb} / \mathrm{GaAs}$. Для $\mathrm{InGaAs} / \mathrm{GaAs}$ гетероструктур была обнаружена зависимость энергий термической активации и сечений захвата акцепторных ловушек дислокации от импульса заполнения - при $V_{r}=0.01 \mathrm{~B}$ и $V_{f}=1.00 \mathrm{~B}$ они стали равными $E_{t}=845$ мэВ и $\sigma_{p}=$ $=1.33 \cdot 10^{-12} \mathrm{~cm}^{2}$ соответственно. Подобная зависимость может быть связана с существованием энергетической зоны в запрещенной зоне. Были произведены оценки времен релаксации концентраций неравновесных носителей при участии в этом процессе глубоких акцепторных ловушек дислокации, которые оказались равными для InGaAs/GaAs гетероструктур $2 \cdot 10^{-10} \mathrm{c}$, a для $\mathrm{GaAsSb} / \mathrm{GaAs}$ гетероструктур $1.5 \cdot 10^{-10} \mathrm{c}$, что существенно меньше, чем для GaAs гомоструктур, $1.6 \cdot 10^{-6} \mathrm{c}$.

Работа выполнена при частичной поддержке Российского фонда фундаментальных исследований (грант РФФИ 16-08-00954-a).

\section{Список литературы}

[1] Ж.И. Алферов, В.И. Корольков, В.Г. Никитин, М.Н. Степанова, Д.Н. Третьяков. Письма ЖТФ, 2 (2), 201 (1976).

[2] Ф.Ю. Солдатенков, В.Г. Данильченко, В.И. Корольков. ФТП, 41 (2), 217 (2007).

[3] В.Г. Данильченко, В.И. Корольков, Ф.Ю. Солдатенков. ФТП, 43 (8), 1093 (2009).

[4] V.A. Kozlov, F.Yu. Soldatenkov, V.G. Danilchenko, V.I. Korolkov, I.L. Shulpina. Proc. 25th Advanced Semiconductor Manufacturing Conf. (Saratoga Springs, USA, May 19-21, 2014) p. 139.

[5] М.М. Соболев, Ф.Ю. Солдатенков, В.А. Козлов. ФТП, 50 (7), 941 (2016).

[6] М.М. Соболев, П.Р. Брунков, С.Г. Конников, М.Н. Степанова, В.Г. Никитин, В.П. Улин, А.Ш. Долбая, Т.Д. Камушадзе, Р.М. Майсурадзе. ФТП, 25 (6), 1058 (1989).

[7] П.Н. Брунков, С. Гайбуллаев, С.Г. Конников, В.Г. Никитин, М.И. Папенцев, М.М. Соболев. ФТП, 25 (2), 338 (1991).

[8] G.M. Martin, A. Mitonneau, A. Mircea. Electron. Lett., 13 (22), 666 (1977).

[9] A. Mitonneau, G.M. Martin, A. Mircea. Electron. Lett., 13 (7), 191 (1977).

[10] V.A. Kaluchov, S.I. Chikichev. Phys. Status. Solidi, 88 (1), K59 (1985).

[11] A.Z. Li, H.K. Kim, J.C. Jeong, D. Wong, T.E. Schlesinger, A.G. Milnes. J. Appl. Phys. 64 (7), 3497 (1988). 
[12] Kohjib Yamada, Kazumi Wada. Inst. Phys. Conf. Ser., 106 (4), 153 (1989).

[13] B.H. Yang, Z.G. Wang, H.J. He, L.Y. Lin. J. Cryst. Growth, 103, 371 (1990).

[14] O. Yastrubchak, T. Wosinski, A. Makosa, T. Figielski, S. Porowski, I. Grzegory, R. Czernecki, P. Perlin. Eur. Phys. J. Appl. Phys., 27, 201 (2004).

[15] T. Wosiński, A. Makosa, J. Raczyńska. Acta Phys. Polon. A, 87 (2), 369 (1995).

[16] В.Г. Данильченко, В.И. Корольков, С.И. Пономарев, Ф.Ю. Солдатенков. ФТП, 45 (4), 524 (2011).

[17] И.Л. Шульпина, В.В. Ратников, В.А. Козлов, Ф.Ю. Солдатенков, В.Е. Войтович. ЖТФ, 84, (10), 149 (2014).

[18] C.O. Thomas, D. Kahng, R.C. Manz. J. Electrochem. Soc., 109, 1055 (1962).

[19] E.S. Yang. J. Appl. Phys., 45 (9), 3801 (1974).

[20] J.P. Donnelly, A.G. Milnes. IEEE Trans. Electron. Dev., ED14 (2), 63 (1967).

[21] М.М. Соболев, А.В. Гитцович, М.И. Папенцев, И.В. Кочнев, Б.С. Явич. ФТП, 26 (10), 1760 (1992).

[22] Д.В. Давыдов, А.Л. Закгейм, Ф.М. Снегов, М.М. Соболев, А.Е. Черняков, А.С. Усиков, Н.М. Шмидт. Письма ЖТФ, 33 (4), 11 (2007).

[23] В.Л. Бонч-Бруевич, С.Г. Калашников. Физика полупроводников (М., Наука, 1977) с. 672.

[24] Г. Матаре. Электроника дефектов в полупроводниках (М., Мир, 1974) с. 464. [Пер. с англ.: Herbert F. Mataré. Defect Electronics in Semiconductors (WileyInterscience a Division of John Wiley and Sons Inc., N.Y.-London-Sydney-Toronto, 1971)].

[25] B. Lax, S.F. Neustadter. J. Appl. Phys., 25 (9), 1148 (1954).

[26] B.R. Gossik. J. Appl. Phys., 27 (7), 905 (1956).

Редактор Г.А. Оганесян

\section{Effect of dislocation deep levels in heteroepitaxial InGaAs/GaAs and GaAsSb/GaAs $p-i-n$ structures on the relaxation time of nonequilibrium carriers}

\author{
M.M. Sobolev, F.Yu. Soldatenkov \\ loffe Institute, \\ 194021 St. Petersburg, Russia
}

\begin{abstract}
We have performed the experimental study of the capacitance-voltage $(C-V)$ characteristics and deep-level transient spectroscopy (DLTS) of $p^{+}-p^{0}-i-n^{0}$ homostructures based on undoped GaAs layers without misfit dislocations and InGaAs/GaAs and $\mathrm{GaAsSb} / \mathrm{GaAs}$ heterostructures with homogeneous network of misfit dislocation grown by liquid-phase epitaxy (LPE). Acceptor defects with deep levels $H L 2$ and $H L 5$ are identified in GaAs epitsxial $p^{0}$ - and $n^{0}$-layers. The electron and hole dislocation deep levels which are denoted as $E D 1$ and $H D 3$ are detected in $\mathrm{InGaAs} / \mathrm{GaAs}$ and $\mathrm{GaAsSb} / \mathrm{GaAs}$ heteristructures. The hole trap parameters calculated from Arrhenius dependencies - thermal activation energy $\left(E_{t}\right)$, carrier cross-section $\left(\sigma_{n}\right)$, concentration $\left(N_{t}\right)$ are $E_{t}=845 \mathrm{meV}$, $\sigma_{p}=1.33 \cdot 10^{-12} \mathrm{~cm}^{2}, N_{t}=3.80 \cdot 10^{14} \mathrm{~cm}^{-3}$ for InGaAs/GaAs and $E_{t}=848 \mathrm{meV}, \sigma_{p}=2.73 \cdot 10^{-12} \mathrm{~cm}^{2}, N_{t}=2.40 \cdot 10^{14} \mathrm{~cm}^{-3}$ for $\mathrm{GaAsSb} / \mathrm{GaAs}$ heterostructures. The estimation of concentration relaxation times for nonequilibrium carries in presence of dislocations associated deep acceptor traps were obtained. They are $2 \cdot 10^{-10} \mathrm{~s}$ and $1.5 \cdot 10^{-10} \mathrm{~s}$ in $\mathrm{InGaAs} / \mathrm{GaAs}$ and $\mathrm{GaAsSb} / \mathrm{GaAs}$ heterostructures respectively, and $1.6 \cdot 10^{-6} \mathrm{~s}$ in GaAs homostructures.
\end{abstract}

